\title{
PREVALENCE AND PROGNOSTIC VALUE OF GERIATRIC SYNDROMES IN ELDERLY PATIENTS IN INTERMEDIATE GERIATRIC REHABILITATION UNITS
}

\author{
M. Serra-Prat ${ }^{1}$, E. Martínez-Suárez'2, R. Cristofol Allue 2 , S.Santaeugènia ${ }^{3}$, M. Roqué ${ }^{4}$, A. Salvà ${ }^{4}$ \\ and the XARESS group
}

\begin{abstract}
Background: The prevalence and prognostic value of geriatric syndromes in geriatric rehabilitation units is poorly understood. Objective: To determine the prevalence of geriatric syndromes in intermediate geriatric rehabilitation units and evaluate associations with outcomes and death during admission. Methods: Observational, longitudinal study of patients admitted to 10 intermediate geriatric rehabilitation units in 2015. Admission-to-discharge data were collected retrospectively from a shared minimum data set (CMBD-RSS). The geriatric syndromes considered were dementia, depression, immobility, urinary incontinence, faecal incontinence, instability, insomnia, acute confusional state, terminal illness and pressure ulcers. The main outcome measures were functional status on admission (assessed using the Resource Utilization Group Activities of Daily Living Scale), functional improvement between admission and discharge, length of stay and death during admission. Results: We analysed 5619 patients (mean age 80.2 years; $57.1 \%$ women). The mean number of syndromes was 2.3 . The most prevalent syndromes were urinary incontinence $(62 \%)$, dementia $(35 \%)$, faecal incontinence $(35 \%)$ and immobility $(26 \%)$. The presence of each geriatric syndrome increased the risk of functional impairment at discharge (except in the case of insomnia) and of death during admission (except in the case of instability syndrome). Conclusions: Geriatric syndromes are very prevalent in intermediate geriatric rehabilitation units and indicate a lower probability of functional recovery and a greater probability of death during admission.
\end{abstract}

Key words: Geriatric syndrome, intermediate geriatric rehabilitation unit, mortality, functional capacity.

\section{Introduction}

Ageing produces physiological changes and a decrease in the functional reserve of different organs and systems that favours the appearance of geriatric syndromes such as dementia, depression, insomnia, acute confusional state (delirium), falls, pressure ulcers and frailty $(1,2)$. Geriatric rehabilitation units in intermediate or subacute care facilities offer subacute and postacute care to elderly patients. These patients need treatment or continuous clinical supervision to recover as much autonomy as possible so that they are sufficiently prepared to return to their social and family life (3). They typically have advanced chronic disease and need to complete medical treatment or rehabilitation before they can be discharged (4). The presence of geriatric syndromes in this

1. Research Unit. Consorci Sanitari del Maresme, Barcelona, Spain; 2. Geriatric Service. Consorci Sanitari del Maresme, Barcelona, Spain; 3. Programa de Prevenció i Atenció a la Cronicitat, Health Department, Catalan Government, Barcelona, Spain; 4. Fundació Salut i Envelliment UAB. Universitat Autònoma de Barcelona, Barcelona, Spain.

Corresponding Author: Mateu Serra-Prat, Research Unit, Hospital de Mataró, Carretera de Cirera s/n, 08304 Mataró, Tel. 9374177 30, e-mail: mserra@csdm.cat population could be an indicator of clinical complexity and poorer prognosis. Detection on admission may help predict functional decline and the risk of unfavourable outcomes, facilitating early planning of care (according to the complexity of each case), length of stay and discharge destination (e.g., home or nursing home) (5). Research in this area, however, is scarce and little is known about the prevalence or prognostic value of geriatric syndromes in this setting (6).

The aims of this study were to determine the prevalence of geriatric syndromes in intermediate geriatric rehabilitation units and to evaluate their association with length of stay, discharge destination, and functional improvement or death during admission.

\section{Material and methods}

\section{Design and study population}

We conducted an observational, longitudinal study of patients in geriatric rehabilitation units at 10 intermediate care facilities with follow-up data from admission to discharge. The 10 facilities are all part of the Catalan 
PREVALENCE OF UNDER NUTRITION AND ASSOCIATED FACTORS AMONG PEOPLE OF OLD AGE IN DEBRE MARKOS TOWN, NORTHWEST ETHIOPIA, 2015.

social health research network XARESS. The data were collected retrospectively from a shared minimum data set (CMBD-RSS). The data collection was coded and anonymized. Only patients with complete stays, defined as admission and discharge in 2015, were included. When there was more than one complete stay in this period, the first stay was selected.

\section{Study variables}

We applied a previously described methodology (6) to estimate the prevalence and number of geriatric syndromes from data in the CMBD-RSS. A patient was considered to have conditions as follows: dementia, when there was mention of recent or distant memory problems or moderately or severely deteriorated cognitive capacity regarding daily decision-making; depression, when there was mention of tears, expressions of sadness, pain, worry or distress or frequent withdrawal from activities of interest (5-7 days a week); immobility, when there was mention of a need for help or total dependence when in bed; urinary incontinence, when there was mention of occasional, frequent or total bladder incontinence or use of a urinary catheter, diapers or compresses; faecal incontinence, when there was mention of occasional, frequent or total faecal incontinence; instability syndrome, when there was mention of partial or total dependence on assistance with walking around a room or hallway; insomnia, when there was mention of insomnia or a change in regular sleep patterns 5-7 nights a week; acute confusional state, when there was mention of signs of delirium; terminal illness, when there was mention of an end-stage disease; and pressure ulcers, when there was mention of a pressure ulcer of any severity (3).

The main outcome measures were functional capacity on admission, functional improvement on discharge, length of stay, discharge destination and death during admission. Functional capacity was assessed using the Resource Utilization Group's Activities of Daily Living (ADL) scale (7), which rates the ability to perform nine ADLs according to whether the person is dependent on help, requires assistance, requires supervision or is independent, with higher scores indicating greater functional impairment. ADLs were classified as dependence-ADLs when the patient was coded as being dependent or requiring assistance, and independenceADLs when the patient was coded as being independent or requiring supervision. The nine ADLs included in the CMBD-RSS were considered. An individual could have anywhere between zero and nine dependence-ADLs on admission and on discharge. Functional improvement was defined as a reduction of one or more in the number of dependence-ADLs between admission and discharge. Patients with no dependence-ADLs on admission and discharge were also included in the functional improvement group. Patients who showed no reduction or change in the number of dependence-ADLs were not considered to have achieved functional improvement.

\section{Data analysis}

We estimated the prevalence of each geriatric syndrome and the mean number of geriatric syndromes per patient at admission and calculated the percentages of patients who showed functional improvement and who died during admission. We also evaluated the associations between each geriatric syndrome and the main outcome measures (ADL score on admission, discharge destination, mean length of stay, functional improvement and death). The t-test was used to compare mean length of stay and mean ADL scores between patients with and without each syndrome. Categorical variables (discharge destination, functional improvement [yes/no] and death) were compared using the chi-square test. The effect of each geriatric syndrome on functional improvement or death was assessed by logistic regression analysis, with odds ratios calculated and adjusted for age, sex and care facility. Finally, multivariate logistic regression analysis was performed to identify the best predictors of functional improvement and death during admission. The multivariate models included geriatric syndromes with an odds ratio of greater than 2 in the univariate analysis, in addition to the number of geriatric syndromes and dependence-ADLs on admission, age, sex and care facility. Statistical significance was established at a p-value of less than 0.05 .

\section{Results}

A total of 5619 patients with complete stays during 2015 were analysed. The mean (SD) age of the sample was 80.2 (10.6) years and $57.1 \%$ were women. The overall prevalence of geriatric syndromes was as follows: dementia $35.3 \%$, depression $16.7 \%$, immobility $26.0 \%$, urinary incontinence $62.0 \%$, faecal incontinence $35.5 \%$, instability $15.1 \%$, insomnia $11.4 \%$, acute confusional state $3.9 \%$, terminal illness $5.2 \%$, and pressure ulcers $11.5 \%$. The mean number of syndromes per patient was 2.3 (1.8) and the median was 2 (range, 0-9). There were notable differences in the prevalence of geriatric syndromes between care facilities, with the mean in each one ranging from 1.2 to 3.5. Nevertheless, all the facilities had patients with no geriatric syndromes and some had patients with as many as five.

Table 1 shows the prevalence of geriatric syndromes on admission according to discharge destination. Not counting the patients who died during admission, the highest prevalence rates were largely observed in patients discharged to a nursing home or another care facility. Patients discharged to their place of usual residence had a mean of 1.9 (median 2) syndromes compared with 3.1 (median 3) syndromes for those discharged to a nursing home and 3.9 (median 4) syndromes for those discharged to another care facility. Table 2 shows that geriatric 
Table 1

Prevalence of geriatric syndromes on admission to an intermediate care rehabilitation unit according to discharge destination and death during admission

\begin{tabular}{|c|c|c|c|c|c|}
\hline Geriatric syndrome & Place of usual residence & Hospital & Intermediate rehabilitation unit & Nursing home & Death during admission \\
\hline Dementia & $28.5 \%$ & $27.3 \%$ & $43.2 \%$ & $62.4 \%$ & $60.3 \%$ \\
\hline Depression & $14.8 \%$ & $16.0 \%$ & $16.9 \%$ & $23.6 \%$ & $23.8 \%$ \\
\hline Immobility & $19.2 \%$ & $25.7 \%$ & $40.2 \%$ & $36.9 \%$ & $48.2 \%$ \\
\hline Urinary incontinence & $55.2 \%$ & $60.0 \%$ & $72.0 \%$ & $84.3 \%$ & $81.7 \%$ \\
\hline Faecal incontinence & $26.5 \%$ & $32.4 \%$ & $49.6 \%$ & $57.7 \%$ & $66.7 \%$ \\
\hline Instability & $14.9 \%$ & $13.6 \%$ & $13.9 \%$ & $18.5 \%$ & $17.0 \%$ \\
\hline Insomnia & $10.5 \%$ & $7.4 \%$ & $13.0 \%$ & $16.6 \%$ & $16.7 \%$ \\
\hline Acute confusional state & $2.7 \%$ & $1.9 \%$ & $3.7 \%$ & $9.3 \%$ & $9.9 \%$ \\
\hline Terminal illness & $1.8 \%$ & $5.5 \%$ & $8.8 \%$ & $1.6 \%$ & $26.7 \%$ \\
\hline Pressure ulcers & $8.3 \%$ & $15.5 \%$ & $18.4 \%$ & $11.7 \%$ & $21.3 \%$ \\
\hline
\end{tabular}

Table 2

Functional capacity on admission and length of stay according to the presence or absence of geriatric syndromes

\begin{tabular}{|c|c|c|c|c|c|c|}
\hline \multirow[t]{3}{*}{ Geriatric syndrome } & \multicolumn{3}{|c|}{ Functional capacity (activities of daily living score) } & \multicolumn{3}{|c|}{ Length of stay (days) } \\
\hline & \multicolumn{2}{|c|}{ G. Syndrome } & \multirow[t]{2}{*}{$\mathbf{P}^{*}$} & \multicolumn{2}{|c|}{ G. Syndrome } & \multirow[t]{2}{*}{$\mathbf{P}^{*}$} \\
\hline & Yes & No & & Yes & No & \\
\hline Dementia & 12.8 & 9.9 & $<0.001$ & 36.9 & 35.9 & 0.130 \\
\hline Depression & 11.4 & 10.9 & 0.020 & 36.5 & 36.2 & 0.771 \\
\hline Immobility & 16.4 & 8.8 & $<0.001$ & 37.9 & 35.6 & 0.003 \\
\hline Urinary incontinence & 12.7 & 8.3 & $<0.001$ & 36.3 & 35.9 & 0.529 \\
\hline Faecal incontinence & 14.2 & 9.4 & $<0.001$ & 36.3 & 36.1 & 0.715 \\
\hline Instability & 14.0 & 10.4 & $<0.001$ & 38.7 & 35.7 & $<0.001$ \\
\hline Insomnia & 12.3 & 10.8 & $<0.001$ & 36.3 & 36.2 & 0.975 \\
\hline Acute confusional state & 13.5 & 10.8 & $<0.001$ & 32.6 & 36.3 & 0.020 \\
\hline Terminal illness & 12.0 & 10.9 & $<0.001$ & 30.1 & 36.5 & $<0.001$ \\
\hline Pressure ulcers & 12.6 & 10.8 & $<0.001$ & 37.6 & 36.0 & 0.085 \\
\hline
\end{tabular}

${ }^{*}$ P-values correspond to t-test

syndromes were associated with greater functional impairment on admission and, with the exception of acute confusional state and terminal illness, a longer mean stay.

Overall, $49.2 \%$ of patients experienced an improvement in functional capacity between admission and discharge, $46.7 \%$ experienced no change and $4.1 \%$ experienced deterioration. The number of geriatric syndromes on admission was negatively correlated with the number of ADLs for which an improvement was observed during admission $(\mathrm{r}=-0.21, \mathrm{p}<0.001)$.

Table 3 shows the association between each geriatric syndrome and functional improvement or death during admission. After adjustment for age, sex and care facility, in almost all cases, the presence of a geriatric syndrome increased the risk of functional impairment (with the exception of insomnia) and of death during admission (with the exception of instability).
Table 4 shows the multivariate analysis results for the independent effect of each geriatric syndrome on functional improvement and death during admission, after adjustment for the presence and number of other geriatric syndromes, the number of dependence-ADLs on admission, age, sex and care facility. Although not highly prevalent, acute confusional state was the strongest predictor of an absence of functional improvement. After terminal illness, faecal incontinence was the strongest predictor of death during admission.

\section{Discussion}

Our results point to a high prevalence of geriatric syndromes in intermediate rehabilitation units, with a mean of 2.3 syndromes per patient. The most common syndrome was urinary incontinence, followed by faecal incontinence, dementia and immobility. Our results also 
PREVALENCE OF UNDER NUTRITION AND ASSOCIATED FACTORS AMONG PEOPLE OF OLD AGE IN DEBRE MARKOS TOWN, NORTHWEST ETHIOPIA, 2015.

Table 3

Association between geriatric syndromes and functional improvement or death during admission

\begin{tabular}{|c|c|c|c|c|c|c|c|c|c|c|}
\hline \multirow[t]{2}{*}{ Geriatric Syndrome (GS) } & \multicolumn{5}{|c|}{$\%$ of patients with improved functional capacity } & \multicolumn{5}{|c|}{$\%$ of deaths during admission } \\
\hline & GS present & GS not present & p & $\mathrm{OR}^{*}$ & p & GS present & GS not present & p & $\mathrm{OR}^{*}$ & $\mathrm{p}$ \\
\hline Dementia & $40.2 \%$ & $62.5 \%$ & $<0.001$ & 2.64 & $<0.001$ & $12.5 \%$ & $4.6 \%$ & 0.517 & 2.36 & $<0.001$ \\
\hline Depression & $50.6 \%$ & $55.4 \%$ & 0.003 & 1.27 & 0.003 & $17.9 \%$ & $4.9 \%$ & 0.213 & 1.65 & $<0.001$ \\
\hline Immobility & $33.9 \%$ & $61.9 \%$ & $<0.001$ & 3.45 & $<0.001$ & $10.8 \%$ & $9.3 \%$ & $<0.001$ & 2.95 & $<0.001$ \\
\hline Urinary incontinence & $47.0 \%$ & $67.0 \%$ & $<0.001$ & 2.18 & $<0.001$ & $13.8 \%$ & $8.8 \%$ & $<0.001$ & 2.33 & $<0.001$ \\
\hline Faecal incontinence & $38.0 \%$ & $63.7 \%$ & $<0.001$ & 2.80 & $<0.001$ & $24.3 \%$ & $8.9 \%$ & $<0.001$ & 3.68 & $<0.001$ \\
\hline Instability & $47.4 \%$ & $55.9 \%$ & 0.155 & 1.65 & $<0.001$ & $49.0 \%$ & $7.4 \%$ & 0.666 & 0.97 & 0.837 \\
\hline Insomnia & $52.0 \%$ & $55.0 \%$ & 0.009 & 1.11 & 0.265 & $17.6 \%$ & $8.5 \%$ & 0.591 & 1.34 & 0.026 \\
\hline Acute confusional syndrome & $33.0 \%$ & $55.5 \%$ & 0.081 & 2.19 & $<0.001$ & $18.5 \%$ & $8.7 \%$ & 0.707 & 2.29 & $<0.001$ \\
\hline Terminal illness & $20.9 \%$ & $56.4 \%$ & 0.001 & 4.82 & $<0.001$ & $12.5 \%$ & $4.6 \%$ & $<0.001$ & 18.2 & $<0.001$ \\
\hline Pressure ulcers & $38.4 \%$ & $56.7 \%$ & 0.002 & 2.04 & $<0.001$ & $17.9 \%$ & $4.9 \%$ & $<0.001$ & 2.31 & $<0.001$ \\
\hline
\end{tabular}

*Odds ratio adjusted for age, sex and intermediate care facility.

show that presence of geriatric syndromes was associated with a poorer prognosis in the form of poorer functional recovery, longer stay, a greater risk of death during admission and a lower probability of being discharged to the home.

Very few studies have evaluated the prevalence of geriatric syndromes in intermediate facilities or, more specifically, in geriatric rehabilitation units (8). Most studies have analysed institutionalized elderly patients in acute care hospitals - a profile of patient that differs somewhat from that of patients in intermediate care facilities. The prevalence rates for depression, pressure ulcers and cognitive impairment are similar to those reported for hospitalized elderly patients $(9,10)$. The fact that higher rates were observed for immobility, urinary incontinence and faecal incontinence $(9,11)$ can probably be explained by the strong association between these three conditions and frailty $(12,13)$, as frail elderly people are more likely to be referred to a rehabilitation unit. The prevalence rates observed for acute confusional state and insomnia were lower than those reported for elderly patients in acute care hospitals $(14,15)$, indicating that these syndromes are more common in the acute care setting. The rates for the different geriatric syndromes analysed were very similar to those reported for the same care facilities in the XARESS network in 2014 (6).

Geriatric syndromes complicate care and indicate a poor prognosis, so systematic assessment is imperative in ensuring adequate management and planning (5, 16, 17). In all cases, we found geriatric syndromes to be associated with a poorer functional status on admission and a lower probability of improvement during admission. This association was most evident for terminal illness, but it was also strong for immobility, dementia and faecal incontinence. In addition, patients with immobility and instability syndrome had longer stays, probably because their advanced age and greater degree of functional dependence reduced the likelihood of being discharged to their place of usual residence.
Our findings also show that the presence of geriatric syndromes influenced discharge destination. Patients with fewer syndromes were more likely to be discharged to their place of usual residence than to a nursing home or another intermediate care facility. Overall, patients with geriatric syndromes were less likely to achieve functional recovery and were more likely to have longer stays, be transferred to another care facility or die during admission. Like terminal illness, both faecal incontinence and immobility were strong predictors of death during admission. The above findings suggest a need to establish suitable prevention, diagnosis and treatment strategies aimed at improving functional status and survival in patients with geriatric syndromes. Diagnosis and treatment requires a multidisciplinary approach based on a comprehensive geriatric assessment (17). Frailty is characterized by a decrease in the functional reserves of different organs and systems that leads to a state of vulnerability to external aggressions or stressors (18). Multiple studies have identified frailty as a risk factor for falls, disability, dependence, institutionalization and even death (19). This syndrome has also been found to be closely associated with other geriatric syndromes $(13,20)$, which would explain its prognostic value.

We were unable to assess frailty in our sample and so could not adjust for its effect on the different syndromes. Our study, nonetheless, shows that most of the geriatric syndromes analysed - most especially, immobility, faecal incontinence, terminal illness and pressure ulcers - exerted an independent effect on functional impairment and mortality during admission. The analysis shows that the higher the number of dependence-ADLs on admission, the more likely the patient was to present functional improvement. This apparently paradoxical effect can be explained by the fact that patients with few dependence-ADLs on admission had less room for improvement than patients with more dependence-ADLs, who were more likely to show improvement in at least one ADL. 
Table 4

Independent effect of geriatric syndromes on the absence of functional improvement and death during admission

\begin{tabular}{|c|c|c|c|c|}
\hline & No functional improvement & Death during admission & & \\
\hline & OR & $\mathrm{p}$ & OR & $\mathrm{p}$ \\
\hline Dementia & 1.69 & $<0.001$ & 1.38 & 0.034 \\
\hline Immobility & 2.30 & $<0.001$ & 1.44 & 0.017 \\
\hline Urinaryincontinence & 1.16 & 0.091 & 0.90 & 0.572 \\
\hline Faecal incontinence & 1.50 & $<0.001$ & 2.01 & $<0.001$ \\
\hline Acute confusional state & 3.84 & $<0.001$ & 1.29 & 0.266 \\
\hline Terminal illness & 1.52 & $<0.001$ & 14.2 & $<0.001$ \\
\hline Pressureulcers & 1.10 & 0.025 & 1.57 & 0.004 \\
\hline Malnutrition & --- & --- & 1.40 & 0.061 \\
\hline Number of geriatric syndromes & 1.69 & $<0.001$ & 1.08 & 0.297 \\
\hline Number of dependence-ADLs on admission & 0.95 & 0.001 & 1.05 & 0.171 \\
\hline
\end{tabular}

ADLs, activities of daily living; OR, odds ratio.

Very few studies have analysed geriatric syndromes in subacute care facilities or, more specifically, in geriatric rehabilitation units (21). Studies of this nature are of great interest, however, given the distinctive characteristics of patients in these settings. A major strength of our study is that the analysis of a large sample of geriatric patients from 10 intermediate care facilities enhances the external validity of our findings and ensures sufficient statistical power to estimate the impact of geriatric syndromes. As for limitations, the most important is related to the quality of the administrative data in the CMBD-RSS minimum data set, as the variability observed between facilities suggests that certain geriatric syndromes may have been underdiagnosed in some units.

In conclusion, geriatric syndromes are very prevalent in patients admitted to geriatric rehabilitation units and their presence is predictive of a lower probability of functional recovery and a greater probability of death during admission. Assessment and early diagnosis of these syndromes could help in planning care and effective use of health and social resources. More studies, however, are needed in to evaluate the effectiveness of such interventions.

XARESS group: Mireia Bosch, Charo Casas, Laura Coll, Dolors Cubí, Joan Conill, Benito Fontecha, Esther Jovell, María Teresa Molins, Antoni Salvà, Jauma Sanahuja, Pau Sanchez, Sebastià Santaeugènia and Mateu Serra-Prat.

Conflict of interest: The authors declare that they have no conflict of interest in relation with this study.

Ethical Standard: The study was carried out respecting all applicable confidenciality rules.

\section{References}

1. Inouye SK, Studenski S, Tinetti ME, Kuchel GA. Geriatric syndromes: Clinical, research and policy implications of a core geriatric concept. J Am Geriatr Soc. 2007; 55(5): 780-791.

2. Carlson C, Merel SE, Yukawa M. Geriatric syndromes and geriatric assessment for the generalist.Med Clin North Am. 2015;99(2):263-79.

3. Santaeugènia SJ, Garcia-Lázaro M. Nuevo Modelo de atención integrada orientada a ancianos ingresados en Unidades de Atención Intermedia en
Cataluña: protocolo de una estudio cuasi experimental. Rev Esp Geriatr Gerontol. 2017;52(4): 201-208.

4. Salva A et al. Descripción del perfil de complejidad de los pacientes admitidos en unidades sociosanitarias de larga estancia entre los años 2003 y 2009. RevEspGeriatGerontol. 2014;49.59-64.

5. Senn N, Monod S. Development of a Comprehensive Approach for the Early Diagnosis of Geriatric Syndromes in General Practice. Front Med (Lausanne). 2015;2:78. doi: 10.3389/ fmed.2015.00078. eCollection 2015.

6. Santaeugènia SJ, Roqué M, Sánchez P, Salvà A. Complejidad y prevalencia de síndromes geriátricos de los pacientes atendidos en unidades sociosanitarias en Cataluña. Estudio multicéntrico del proyecto XARES. Rev Esp Geriatr Gerontol 2018. https: / / doi.org/10.1016/j.regg2018.10.006

7. Romeo S, Gala B, Gómez E. Uso de escalas de valoración en el proyecto de ley de promoción de la autonomía personal y de atención a las personas dependientes. Index Enferm 2006; 15 (54).

8. Wang H, Niewczyk P, DiVita M, et al: Impact of pressure ulcers on outcomes in inpatient rehabilitation facilities. Am J Phys Med Rehabil 2014;93:207-16.

9. Garcia T, López JA, Villalobos JA, d'Hyver C. Prevalencia de sindromes geriátricos en ancianos hospitalizados. Med interna Mex 2006; 22(5): 369-74.

10. McCusker J, Cole M, Dufouil C. The prevalence and correlates of major and minor depression in older medical inpatients. J Am Geriatr Soc 2005; 53: 134453.

11. Remes JM, Sáenz P, Riaño D, et al. Incontinencia fecal en adultos mayores. Rev Investigación Clínica 2004; 56(1): 21-6.

12. M Cabré, L Elias, M García, E Palomera, M Serra-Prat. Hospitalizaciones evitables por reacciones adversas medicamentos en una unidad geriátrica de agudos. Análisis de 3292 pacientes. Med Clin (Barc), 2018;150(6), 209-214

13. Serra-Prat M, Papiol M, Vico J, Palomera E, Sist X, Cabré M. Factors associated with frailty in community-dwelling elderly population. A cross-sectional study. Eur Geriatric Med 2016; 7 (6): 531-7.

14. Chávez-Delgado ME, Virgen-Enciso M, Pérez-Guzman, Celis-de la Rosa M, Castro-Castañeda S. Delirio en pacientes hospitalizados. Rev Med Inst Mex Seguro Soc 2007; 45 (4): 321-8.

15. Pi-Figueras M, Aguilera A, Arellano M, Miralles R, Garcia-Caselles P, Torres R, Cervera AM. Prevalence of delirium in a geriatric convalescence hospitalization unit: patient's clinical characteristics and risk precipitating factor analysis. Arch Gerontol Geriatr Suppl. 2004;(9):333-7.

16. Carlson C, Merel SE, Yukawa M.Geriatric syndromes and geriatric assessment for the generalist. Med Clin North Am2015;99(2):263-79.

17. Carlson C, Merel SE, Yukawa M.Geriatric syndromes and geriatric assessment for the generalist. Med Clin North Am2015;99(2):263-79.

18. Morley J, Vellas B, van Kan GA, Anker SD, Bauer JM, Bernabei R, et al. Frailty consensus: a call to action. Journal of the American Medical Directors Association 2013; 14: 392-7.

19. Vermeiren S, Vella-Azzopardi R, Beckwée D, Habbig AK, Scafoglieri A Jansen B, Bautmans I; Gerontopole Brussels Study group. Frailty and the Prediction of Negative Health Outcomes: A Meta-Analysis.J Am Med DirAssoc2016;17(12):1163.e1-1163.e17

20. Tze Pin Ng, Liang Feng, Ma Shwe Zin Nyunt, Anis Larbi, Keng Bee Yap. Frailty in older persons: multisystem risk factors and the frailty risk index (FRI). JAMDA 2014; 15: 635-42.

21. Esperanza A. et al Evaluation of functional improvement in older patients with cognitive impairment, depression and/or delirium admitted to a geriatric convalescence hospitalization unit. Arch Gerontol Geriatr 2004 (Suppl 9); 149153. 